\title{
Cytokine producing ability of peripheral blood cells from COVID-19 patients after unspecific in vitro stimulation
}

\author{
Snezana Zivancevic-Simonovic ${ }^{1}$ (1) $\cdot$ Danijela Jovanovic $^{2,3}$ (1) $\cdot$ Vojislav Cupurdija $^{2,3} \cdot$ Olivera Milosevic-Djordjevic $^{4}[$ [ \\ Marijana Stanojevic ${ }^{3,5}$ - Milos Marinkovic ${ }^{3} \cdot$ Nebojsa Igrutinovic $^{3} \cdot$ Ivan Stanojevic ${ }^{6} \cdot$ Danilo Vojvodic $^{6} \mathbb{}$. $^{\circ}$ \\ Olgica Mihaljevic ${ }^{1}[$
}

Received: 24 October 2021 / Revised: 29 December 2021 / Accepted: 29 January 2022 / Published online: 14 February 2022

(c) The Author(s), under exclusive licence to Springer Nature Switzerland AG 2022

\begin{abstract}
Objective and design Perturbations of peripheral $\mathrm{T}$ cell homeostasis and dysregulation of the immune response to SARSCoV-2, especially in severely ill patients, were observed. The aim of this study was to analyze the cytokine producing ability of peripheral blood cells from severely ill COVID-19 patients upon non-specific in vitro stimulation with phytohemagglutinin (PHA). Possible associations of cytokine levels with patients' age and gender, glucocorticosteroid therapy, as well as the trend of the inflammatory process at the time of sampling (increased or decreased) were also analyzed.

Subjects and methods The study included 23 COVID-19 patients and 17 healthy control subjects. The concentrations of selected Th1/Th2/Th9/Th17/Th22 cytokines were determined using a multi-analyte flow assay kit.

Results Our results showed that peripheral blood cells from severely ill COVID-19 patients had a much reduced ability to produce cytokines in comparison to healthy controls. When inflammation was raised, blood cells produced more IL-6 and IL-17, which led to increases of some Th17/Th1 and Th17/Th2 ratios, skewing towards the Th17 type of response. The methylprednisolone used in the treatment of patients with COVID-19 influences the production of several cytokines in dose dependent manner.

Conclusion Our results indicate that the stage of the inflammatory process at the time of sampling and the dose of the applied glucocorticosteroid therapy might influence cytokine producing ability upon non-specific stimulation of T cells in vitro.
\end{abstract}

Keywords Cytokine $\cdot$ COVID-19 $\cdot$ Phytohemagglutinin $\cdot$ In vitro $\cdot$ Inflammation $\cdot$ Glucocorticosteroid

\section{Introduction}

Responsible Editor: Anatoliy Kubyshkin.

Snezana Zivancevic-Simonovic snezana@medf.kg.ac.rs

1 Department of Pathophysiology, Faculty of Medical Sciences, University of Kragujevac, Svetozara Markovica 69, 34000 Kragujevac, Serbia

2 Department of Internal Medicine, Faculty of Medical Sciences, University of Kragujevac, Kragujevac, Serbia

3 University Clinical Center Kragujevac, Kragujevac, Serbia

4 Department of Genetics, Faculty of Medical Sciences, University of Kragujevac, Kragujevac, Serbia

5 Department of Biochemistry, Faculty of Medical Sciences, University of Kragujevac, Kragujevac, Serbia

6 Institute for Medical Research, Military Medical Academy, Belgrade, Serbia
Coronavirus disease 2019 (COVID-19) is caused by severe acquired respiratory syndrome coronavirus 2 (SARSCoV-2). Since late 2019, COVID-19 grew into a pandemic, with millions of infected people. Even though the majority of infected people are asymptomatic or have a mild respiratory illness, approximately $20 \%$ of them become seriously ill and require hospitalization due to pneumonia and/or systemic complications [1]. In some patients, acute respiratory distress syndrome (ARDS) and systemic inflammation accompanied by multiple organ dysfunction syndrome (MODS) occur [2].

SARS-CoV-2 binds to epithelial cells via angiotensinconverting enzyme 2 (ACE2). Then, the host immune system recognizes the whole virus or its surface epitopes, eliciting the innate or adaptive immune response [3-9]. Pathogen recognition receptors (PRRs), mainly Toll-like receptors 
(TLRs) [10], are expressed in immune cells, fibroblasts, and epithelial cells, including type II pneumocytes in the airways. The activation of TLRs leads to the subsequent production of type I IFNs and inflammatory cytokines via the activation of nuclear factor- $\mathrm{KB}(\mathrm{NF}-\kappa \mathrm{B})[9,11]$. These cytokines further recruit neutrophils and monocytes to the site of infection.

Neutrophilia, lymphopenia and an increased neutrophilto-lymphocyte ratio were observed in the peripheral blood of COVID-19 patients [6] as well as perturbations of peripheral $\mathrm{T}$ cell homeostasis and dysregulation of the immune response to SARS-CoV-2, especially in severely ill patients $[3,6,7]$. In the most severe forms of SARS-CoV-2 infection, large numbers of innate and adaptive immune cells become activated and begin to produce pro-inflammatory cytokines, establishing an exacerbated feedback loop of inflammation [12]. The plasma cytokine levels analyzed in the majority of studies indicated the release of very large amounts of proinflammatory, mainly T-helper-1 (Th1) cytokines (IL1, IL-6, IL-12, IFN- $\gamma$, and TNF- $\alpha$ ) [13] and T-helper-17 (Th17) cytokines [3, 14]. Moreover, increased secretion of T-helper-2 (Th2) cytokines (IL-4 and IL-10) were also observed, whereby IL-10 may suppress inflammation [15]. In most severe cases, hyper production leads to a cytokine storm [16-18] with functional exhaustion of both innate and adaptive immune responses [14, 18].

In sepsis and non-infectious SIRS a paradoxically diminished ability of circulating leukocytes to produce cytokines upon ex vivo activation has already been shown [19]. Recently published studies have analyzed cytokine production by blood cells from COVID-19 patients upon non-specific in vitro stimulation with Toll-like receptor $7 / 8$ (TLR 7/8) agonist and anti-CD3 antibody [18], anti-CD3/ anti-CD28 antibodies [10, 16], superantigens, pokeweed mitogen or Concanavalin A [7], but the results are somewhat contradictory. The in vitro production of cytokines from peripheral blood cells of COVID-19 patients upon non-specific activation with phytohemagglutinin (PHA), a mitogenic lectin known to activate $\mathrm{T}$ cells in the presence of monocytes [21], has not been investigated so far. The aim of this study was to determine the cytokine producing ability of peripheral blood cells from severely ill COVID-19 patients in PHA-stimulated whole blood cultures in vitro. We analyzed the secretion of selected Th1/Th2/Th9/Th17/ Th22 cytokines and possible associations of cytokine production with the patients' age and gender as well as dose and duration of glucocorticosteroid therapy and trend of the inflammatory process at the time of sampling (increased or decreased).

\section{Materials and methods}

\section{Patients}

The study population included 23 COVID19 patients (16 males and 7 females) of mean age $60.52 \pm 12.04$ (range 29-83) years. COVID-19 patients were diagnosed according to the World Health Organization's (WHO) interim guidance [22]. All patients were recruited from the Clinic for Lung Diseases of the Clinical Center Kragujevac. Inclusion criteria were as follows: SARS-CoV-2 infection confirmed by real-time polymerase chain reaction (RT-PCR), hospitalization and severe clinical expression of the disease, radiographically proven pneumonia needing supplemental oxygen, but not too advanced to require intubation and mechanical ventilation at the time of blood collection. Besides oxygen therapy, all the patients received antibiotics and methylprednisolone but were not treated with anti-interleukin 6 monoclonal antibody. The studied patients had no autoimmune or allergic disease. Seventeen healthy subjects with a negative antigenic test for coronavirus (11 males and 6 females) of mean age $55.53 \pm 11.75(28-71)$ years were included in the investigation as a control group. The control subjects had no acute or chronic infective, autoimmune, or allergic diseases and were not vaccinated against SARSCoV-2 before enrolment. The study was planned according to ethical guidelines following the Declaration of Helsinki. The institutional review committee approved our protocol (number 01/21-138) according to local biomedical research regulations. All patients and control subjects gave informed consent prior to enrolment in the study.

\section{Whole blood culture}

Whole blood culture was performed as described earlier $[23,24]$ with a few modifications. Heparinized whole blood ( $0.5 \mathrm{ml}$ per subject) was added to $2 \mathrm{ml}$ RPMI based complete medium containing fetal bovine serum, L-glutamine and phytohemagglutinin (PHA) (GIBCO ${ }^{\mathrm{TM}} \mathrm{PB}-\mathrm{MAX}^{\mathrm{TM}}$ karyotyping medium, Invitrogen, California, USA) and incubated at $37^{\circ} \mathrm{C}$ for $48 \mathrm{~h}$. The supernatant was harvested by centrifugation ( $2000 \mathrm{rpm}$ for $12 \mathrm{~min}$ ) and then stored at $-20{ }^{\circ} \mathrm{C}$ until required.

\section{Cytokine measurements}

Cytokines were determined in supernatants obtained from whole blood cultures of COVID-19 patients and control subjects. The supernatant samples were thawed and analyzed using a multi-analyte flow assay kit for human Th1/Th2/Th9/ Th17/Th22 (LEGENDplex ${ }^{\mathrm{TM}}$, Human Th Cytokine Panel 
(13-plex), BioLegend, San Diego, USA) according to the manufacturer's instructions. All samples were acquired and analyzed on a FC500 Beckman Coulter Flow Cytometer. Collected data were examined on the basis of the measured mean fluorescence intensities (MFI) using standard curves constructed with the MFI values for known cytokine concentrations given in the cytokine kit. Assay sensitivities (minimum detectable concentration $+2 \mathrm{SD}$ ) for each cytokine determined in cell culture medium were as follows: IL-2, $1.9 \mathrm{pg} / \mathrm{ml}$; IL-4, $1.7 \mathrm{pg} / \mathrm{ml}$; IL-5, $2.50 \mathrm{pg} / \mathrm{ml}$; IL-6, $2.10 \mathrm{pg} /$ ml; IL-9, 2.7 pg/ml; IL-10, 1.3 pg/ml; IL-13, 1.9 pg/ml; IL17A, $2.6 \mathrm{pg} / \mathrm{ml}$; IL-17F, $1.7 \mathrm{pg} / \mathrm{ml}$; IL-21, 9 pg/ml; IL-22, $2.2 \mathrm{pg} / \mathrm{ml} ; \mathrm{IFN}-\gamma, 2.4 \mathrm{pg} / \mathrm{ml} ; \mathrm{TNF}-\alpha, 1.5 \mathrm{pg} / \mathrm{ml}$.

\section{Statistical analysis}

Statistical analysis was carried out using the commercial SPSS version 18.0 for Windows. The Kolmogorov-Smirnov test was performed to verify the distribution of certain variables. Differences between two independent groups were evaluated by Student's $t$-test (for variables with normal distribution), or the Mann-Whitney test (for variables without normal distribution). The linear correlation test and determination of the Pearson/Spearman coefficient were applied to estimate association between tested variables. The strength of the relationship between several independent variables and a single continuous dependent variable was assessed by multivariate regression test. A probability $(p)$ value less than 0.05 was considered the threshold for statistical significance.

\section{Results}

We analyzed the cytokine producing ability of peripheral blood cells from 23 COVID-19 patients and 17 control subjects. The characteristics of our COVID-19 patients are given in Table 1. There was no correlation between CRP and the number of leucocytes (Spearman $r=0.242, p=0.266$ ), but a statistically significant inverse correlation of CRP levels with the number of lymphocytes in our patient group was observed (Spearman $r=-0.593, p=0.003$ ). A high value for CRP concentration as a marker of inflammation corresponded to a lower lymphocyte count.

\section{Cytokine production in COVID-19 patients and healthy controls}

Cytokine concentrations were measured in the supernatants of 48-h PHA-stimulated whole blood cultures in vitro. The results obtained for the COVID-19 patients and control subjects are shown in Table 2.

The PHA stimulated peripheral blood cells from healthy controls produced significantly more of the following cytokines: IL-2 $(p=0.001)$, IL-4 $(p=0.020)$, IL-6 $(p<0.001)$, IL-10 $(p=0.001)$, IL-13 $(p=0.006)$, IL-17A $(p<0.001)$, IL-17F $(p<0.001)$, IFN- $\gamma(p=0.004)$ and TNF- $\alpha(p=0.007)$. The mean production of the remaining cytokines (IL-5, IL-9, IL-21 and IL-22) tended to be higher in healthy controls than in patients with COVID-19, but the differences did not reach statistical significance. Additionally, we compared the concentration of proinflammatory cytokine IL-6 measured in supernatant from whole blood culture of COVID-19 patients with its concentration in serum samples. Our results showed negative correlation between supernatant and serum values of IL-6 in COVID19 patients, statisticaly not significant (Bivariate correlation test, Spearman $r=-0.244, p=0.262$ ).

Since the nonspecific in vitro stimulation was done by adding $0.5 \mathrm{ml}$ of whole blood into culture medium, our cultures contained different numbers of stimulated cells. A comparison between the counts of white blood cells and its subpopulations in COVID-19 patients and control subjects showed a significantly lower lymphocyte count
Table 1 Hematological and biochemical parameters for 23 COVID-19 patients and 17 control subjects

\begin{tabular}{|c|c|c|c|c|c|}
\hline \multirow[t]{2}{*}{ Parameters } & \multicolumn{2}{|c|}{ COVID-19 patients } & \multicolumn{2}{|c|}{ Control subjects } & \multirow[t]{2}{*}{ Reference range } \\
\hline & $X \pm \mathrm{SD}$ & Range & $X \pm \mathrm{SD}$ & Range & \\
\hline WBC $\left(\times 10^{9} / 1\right)$ & $10.18 \pm 3.33$ & $6.95-21.58$ & $6.7 \pm 1.0$ & $5.3-8.21$ & $3.7-10.0$ \\
\hline Granulocytes $\left(\times 10^{9} / 1\right)$ & $8.38 \pm 3.36$ & $5.09-20.15$ & $3.84 \pm 1.01$ & $2.4-6.27$ & $2.1-6.5$ \\
\hline Lymphocytes $\left(\times 10^{9} / 1\right)$ & $1.10 \pm 0.55$ & $0.42-2.17$ & $2.23 \pm 0.48$ & $1.4-3.1$ & $1.2-3.4$ \\
\hline Monocytes $\left(\times 10^{9} / 1\right)$ & $0.63 \pm 0.39$ & $0.12-1.93$ & $0.58 \pm 0.14$ & $0.4-0.75$ & $0.1-0.8$ \\
\hline AST (IU/l) & $38.91 \pm 27.29$ & $14-117$ & $14.8 \pm 9.2$ & $6-37$ & $0.0-40.0$ \\
\hline LDH (U/l) & $630.95 \pm 145.02$ & $361-826$ & $213.2 \pm 79.2$ & $125-354$ & $220-450$ \\
\hline $\mathrm{CRP}(\mathrm{mg} / \mathrm{l})$ & $63.95 \pm 68.47$ & $5.9-317.7$ & $1.4 \pm 0.4$ & $<1.0-2.0$ & $<5$ \\
\hline IL-6 (pg/ml) & $39.13 \pm 26.48$ & $11.2-111.4$ & n.d. & n.d. & $<6$ \\
\hline PCT (ng/ml) & $0.15 \pm 0.37$ & $0.04-1.78$ & n.d. & n.d. & $0.5-2.0$ \\
\hline
\end{tabular}

$W B C$ white blood cells, $A S T$ aspartate aminotransferase, $L D H$ lactate dehydrogenase, $C R P$ C-reactive protein, $I L-6$ interleukin 6, $P C T$ procalcitonin, $n . d$. not determined 
Table 2 Cytokine concentrations in PHAstimulated whole blood cultures from COVID-19 patients and healthy controls

\begin{tabular}{|c|c|c|c|c|c|}
\hline \multirow[t]{2}{*}{ Cytokine } & \multicolumn{2}{|c|}{ COVID-19 patients $(n=23)$} & \multicolumn{2}{|c|}{ Healthy controls $(n=17)$} & \multirow{2}{*}{$\begin{array}{l}\text { Independent } \\
\text { samples test }\end{array}$} \\
\hline & $X \pm \mathrm{SD}$ & Min-max & $X \pm \mathrm{SD}$ & Min-max & \\
\hline IL-2 & $31.45 \pm 52.18$ & $6.13-248.29$ & $986.43 \pm 1657.8$ & 24.9-5965.02 & $p=0.001$ \\
\hline IL-4 & $49.21 \pm 22.34$ & 28.15-126.51 & $916.97 \pm 1907.9$ & $58.30-7377.8$ & $p=\mathbf{0 . 0 2 0}$ \\
\hline IL-5 & $23.34 \pm 36.64$ & ${ }^{\mathrm{a}} 1.50-178.92$ & $1419.7 \pm 1156.4$ & $152.29-3797.66$ & $p=0.496$ \\
\hline IL-6 & $320.53 \pm 574.12$ & $5.20-2227.0$ & $6494.6 \pm 4319.2$ & $1516-13,980$ & $p<0.001$ \\
\hline IL-9 & $31.93 \pm 5.58$ & $25.87-48.12$ & $2916.6 \pm 4452.3$ & $48.83-13,654.62$ & $p=0.085$ \\
\hline IL-10 & $32.27 \pm 20.64$ & 25.10-125.70 & $579.85 \pm 793.28$ & 36.99-2894.77 & $p=0.001$ \\
\hline IL-13 & $90.86 \pm 200.36$ & 25.59-900.87 & $6828.3 \pm 6553.7$ & $589.17-18,560.28$ & $p=0.006$ \\
\hline IL-17A & $139.2 \pm 557.86$ & b $0.54-2965.48$ & $3466.1 \pm 3683$ & 102.32-11,073.7 & $p<0.001$ \\
\hline IL-17F & $46.33 \pm 89.77$ & $2.0-442.85$ & $409.59 \pm 355.18$ & 11.18-1363.99 & $p<0.001$ \\
\hline IL-21 & $29.73 \pm 13.16$ & $14.18-72.98$ & $102.66 \pm 221.72$ & $11.69-870.52$ & $p=0.543$ \\
\hline IL-22 & $14.01 \pm 24.57$ & $2.78-119.41$ & $827.16 \pm 1226.5$ & $8.76-4622$ & $p=0.077$ \\
\hline IFN- $\gamma$ & $41.15 \pm 26.05$ & 26.02-152.87 & $1562.3 \pm 1641.5$ & 133.89-5610.24 & $p=0.004$ \\
\hline TNF- $\alpha$ & $88 \pm 42.22$ & 48.38-217.88 & $3415.34 \pm 3297$ & $95.78-9465.22$ & $p=0.007$ \\
\hline
\end{tabular}

Significant differences are given in bold, $p<0.05$

IL-2, 1.9 pg/ml; IL-4, 1.7 pg/ml; IL-5, 2.50 pg/ml; IL-6, 2.10 pg/ml; IL-9, 2.7 pg/ml; IL-10, 1.3 pg/ml; IL-13, 1.9 pg/ml; IL-17A, 2.6 pg/ml; IL-17F, 1.7 pg/ml; IL-21, 9 pg/ml; IL-22, 2.2 pg/ml; IFN- $\gamma, 2.4$ pg/ $\mathrm{ml} ; \mathrm{TNF}-\alpha, 1.5 \mathrm{pg} / \mathrm{ml}$

${ }^{\mathrm{a}}$ One sample below detection limit

${ }^{\mathrm{b}}$ Three samples below detection limit $\left(1.10 \pm 0.55\right.$ vs $\left.2.23 \pm 0.47 \times 10^{9} / 1\right)$ (independent samples $T$ test, $p<0.001$ ) in patients with COVID-19, while the count of monocytes was almost identical in the both study groups $\left(0.63 \pm 0.39\right.$ vs $\left.0.59 \pm 0.16 \times 10^{9} / 1\right)($ Mann Whitney test, $p=0.860$ ). Accordingly, the large differences in cytokine concentration shown in Table 2 might have been a consequence of unequal cell numbers and/ or variable capacities of the stimulated cells to produce cytokines. Therefore, the measured values for cytokine concentrations were multiplied by a correction factor for each patient and control subject in order to obtain values corresponding to production by 1000 stimulated lymphocytes, thereby excluding the influence of cell number on cytokine levels measured in the culture medium. The values obtained after correction for cell number are shown in Tables 3, 4 and 5 and Figs. 1a, b and 2.

As shown in Table 3, the statistically significant differences between production of cytokines by COVID-19 patients and controls were confirmed for the majority (IL-2, IL-5, IL-6, IL-13, IL-17A, IL-17F, IL-21, IL-22, TNF- $\alpha$, and IFN- $\gamma$ ). These results indicate that the response of lymphocytes from COVID-19 patients to strong and nonspecific in vitro stimulation with PHA is markedly reduced compared to those from healthy subjects regarding ability to produce cytokines.

Since the large individual variation in cytokine production (in both study groups) was not lost after correction for cell number, we examined the ratios between selected cytokine levels in each subject individually. The mean values of cytokine ratios are shown in Table 4.

Our results did not show a clear shift in production of selected cytokines towards Th1, Th2 or Th17 types. Namely, the ratios of Th1 cytokine levels (IL-2, TNF- $\alpha$, and IFN$\gamma$ ) with IL-13 (which is a Th2 cytokine) were higher in COVID-19 patients than in controls. However, the ratios of Th1 cytokines with IL-4 (also a Th2 type of cytokine) were lower in COVID-19 patients than in control subjects. The ratios of Th1 cytokines with IL-6 (which produce a number of cells, including Th2 lymphocytes), were higher in COVID-19 patients than in controls, reaching statistical significance for IL-2/IL-6 and IFN- $\gamma /$ IL-6. The Th17/ Th1 cytokine ratios and Th17/Th2 cytokine ratios (IL-17A/ IL-2, IL-17A/IFN- $\gamma$, IL-17A/IL-4, IL-17A/IL-10) were significantly lower in COVID-19 patients than in controls or without statistically proven differences (IL-17A/TNF- $\alpha$, IL-17F/IL-2, IL-17F/TNF- $\alpha$, IL-17F/IFN- $\gamma$, IL-17F/IL-4, IL-17F/IL-5).

In this study we analyzed possible associations of cytokine production with gender and age (Fig. 1). Mean concentrations of all cytokines were similar in male and female COVID-19 patients, except for IL-13. Levels of IL-13 $(167.88 \pm 244.49$ vs $57.51 \pm 23.05, p=0.039)$ were higher in males $(n=16)$ than in females $(n=7)$ (Fig. 1a). There were no statistically significant differences in cytokine levels between the subgroup of patients aged under 60 years $(n=11)$ and those older than 60 years $(n=12)$ (Fig. 1b). Also, no correlation of cytokine production with the age of 


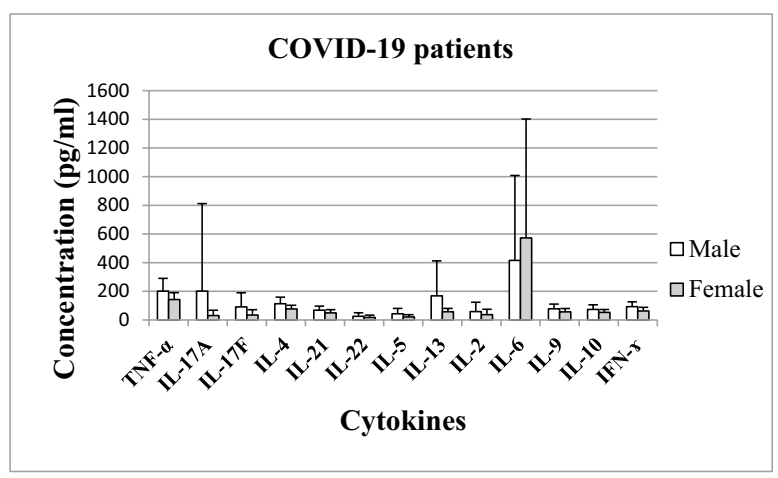

b

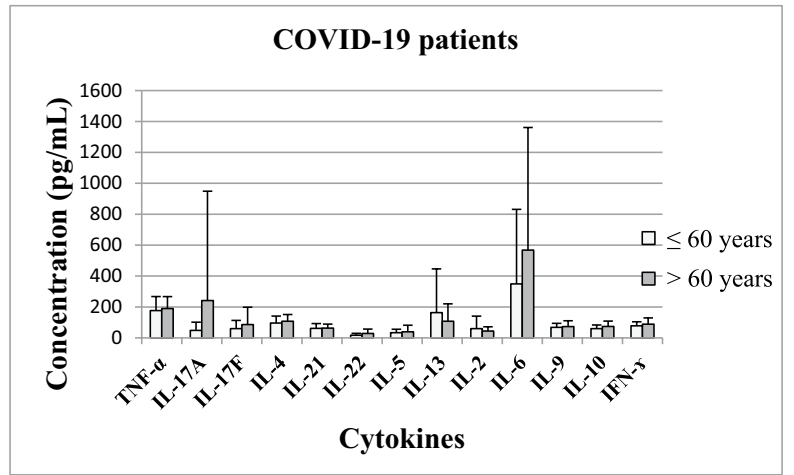

c

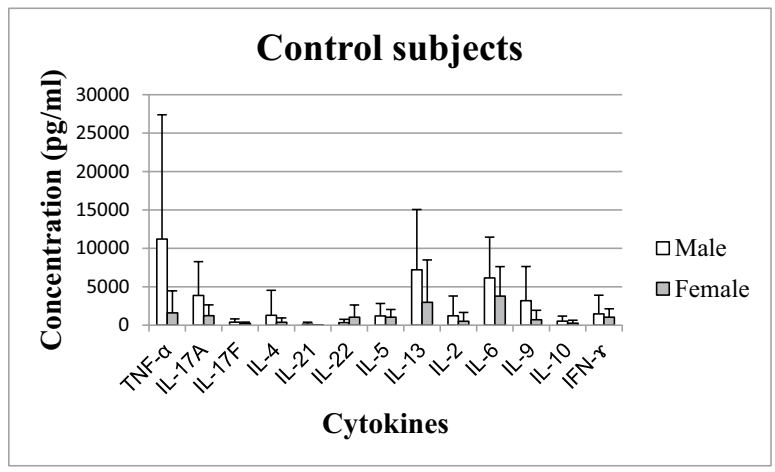

d

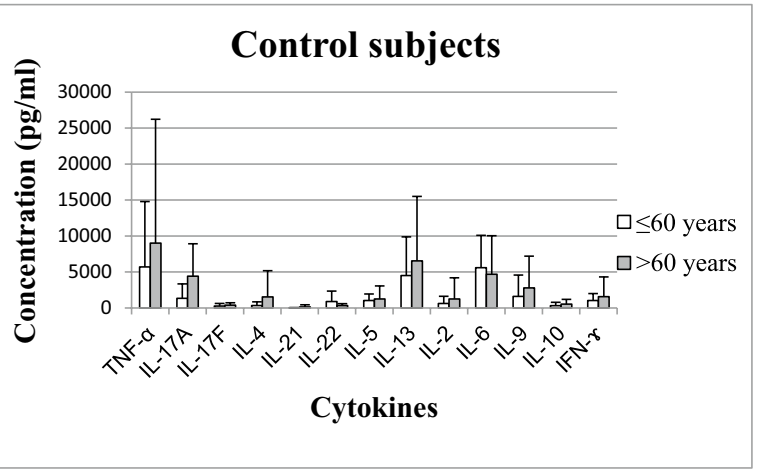

Fig. 1 Cytokine concentrations in PHA-stimulated whole blood cell cultures of COVID-19 patients analyzed in relation to gender (a) and age (b) and control subjects analyzed in relation to gender (c) and age (d)

Table 3 Cytokine concentrations in the supernatants of 48-h PHAstimulated whole blood cells calculated per 1000 in vitro stimulated lymphocytes

\begin{tabular}{lccl}
\hline Cytokine & COVID-19 patients & Control subjects & $\begin{array}{l}p \text { (Mann } \\
\text { Whitney } \\
\text { test })\end{array}$ \\
\hline IL-2 & $\mathbf{5 1 . 8 2} \pm \mathbf{5 8 . 2 5}$ & $\mathbf{9 2 3 . 2 3} \pm \mathbf{2 0 9 3 . 3 2}$ & $\boldsymbol{p}=\mathbf{0 . 0 0 7}$ \\
IL-4 & $102.19 \pm 43.62$ & $908.39 \pm 2510.15$ & $p=0.051$ \\
IL-5 & $\mathbf{3 6 . 6 1} \pm \mathbf{3 3 . 6 1}$ & $\mathbf{1 1 3 8 . 3 8} \pm \mathbf{1 3 6 3 . 7 4}$ & $\boldsymbol{p}<\mathbf{0 . 0 0 1}$ \\
IL-6 & $\mathbf{4 6 3 . 4 6} \pm \mathbf{6 5 7 . 6 7}$ & $\mathbf{5 1 7 4 . 1 5} \pm \mathbf{4 7 7 9 . 6 3}$ & $\boldsymbol{p}<\mathbf{0 . 0 0 1}$ \\
IL-9 & $71.05 \pm 31.68$ & $2165.38 \pm 3645.34$ & $p=0.120$ \\
IL-10 & $67.25 \pm 30.12$ & $417.96 \pm 563.33$ & $p=0.126$ \\
IL-13 & $\mathbf{1 3 4 . 2 9} \pm \mathbf{2 0 8 . 8 0}$ & $\mathbf{5 4 6 6 . 4 3} \pm \mathbf{7 1 1 9 . 0 4}$ & $\boldsymbol{p}<\mathbf{0 . 0 0 1}$ \\
IL-17A & $\mathbf{1 4 9 . 2 0} \pm \mathbf{5 1 1 . 1 3}$ & $\mathbf{2 7 8 4 . 4 9} \pm \mathbf{3 6 6 4 . 0 5}$ & $\boldsymbol{p}<\mathbf{0 . 0 0 1}$ \\
IL-17F & $\mathbf{7 3 . 7 4} \pm \mathbf{8 7 . 9 3}$ & $\mathbf{3 2 8 . 3 2} \pm \mathbf{3 4 1 . 9 4}$ & $\boldsymbol{p}=\mathbf{0 . 0 0 3}$ \\
IL-21 & $\mathbf{6 3 . 3 3} \pm \mathbf{2 7 . 9 0}$ & $\mathbf{8 4 . 7 4} \pm \mathbf{2 0 5 . 8 3}$ & $\boldsymbol{p}=\mathbf{0 . 0 0 9}$ \\
IL-22 & $\mathbf{2 2 . 9 5} \pm \mathbf{2 2 . 6 2}$ & $\mathbf{6 2 7 . 1 0} \pm \mathbf{1 0 8 5 . 0 7}$ & $\boldsymbol{p}=\mathbf{0 . 0 2 2}$ \\
TNF- $\boldsymbol{\alpha}$ & $\mathbf{1 8 3 . 4 8} \pm \mathbf{8 2 . 4 2}$ & $\mathbf{7 2 5 5 . 9 1} \pm \mathbf{1 3 , 1 9 1 . 7 9}$ & $\boldsymbol{p}=\mathbf{0 . 0 0 6}$ \\
IFN- $\boldsymbol{\gamma}$ & $\mathbf{8 3 . 6 3} \pm \mathbf{3 4 . 0 6}$ & $\mathbf{1 2 9 3 . 7 2} \pm \mathbf{1 9 5 4 . 7 4}$ & $\boldsymbol{p}=\mathbf{0 . 0 0 1}$ \\
\hline
\end{tabular}

Significant differences are given in bold, $p<0.05$

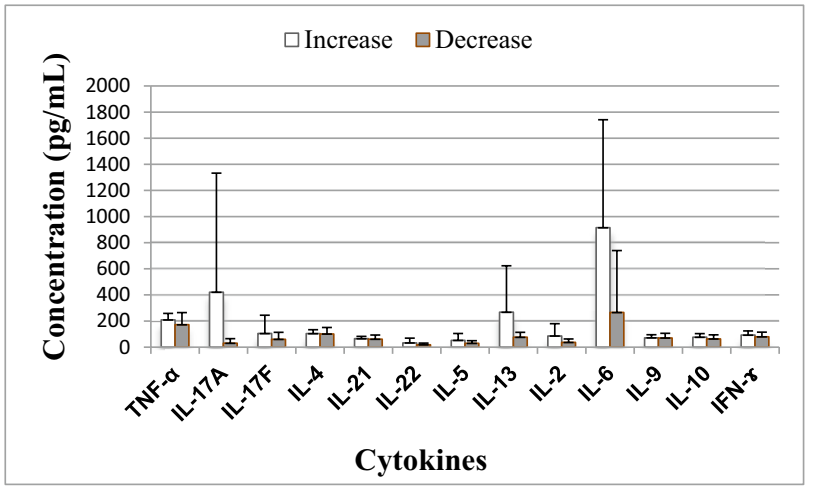

Fig. 2 Cytokine production from PHA-stimulated peripheral blood cells of COVID-19 patients in whom inflammation increased $(n=7)$ or decreased $(n=16)$. Significant differences in IL-17A $(p=0.015)$; significant difference in IL-6 $(p=0.026)$

our patients was observed except for interleukin 22 (Spearman $r=0.417, p=0.048)$. Mean concentrations of all cytokines were similar in male $(n=10)$ and female $(n=7)$ control subjects (Fig. 1c). There were no statistically significant differences in cytokine levels between the subgroup 
Table 4 Mean values $(\mathrm{X} \pm \mathrm{SD})$ of cytokine ratios for COVID-19 patients and healthy controls

\begin{tabular}{lll}
\hline Cytokine ratio & $\begin{array}{l}\text { COVID-19 } \\
\text { patients } \\
(n=23)\end{array}$ & $\begin{array}{l}\text { Control subjects } \\
(n=17)\end{array}$ \\
& $X \pm \mathrm{SD}$ & \\
\hline
\end{tabular}

Th1/IL-6

\begin{tabular}{|c|c|c|c|}
\hline IL-2/IL-6 & $0.33 \pm 0.35$ & $0.09 \pm 0.12$ & $p=0.001$ \\
\hline TNF- $\alpha /$ IL-6 & $1.97 \pm 3.29$ & $0.86 \pm 1.06$ & $p=0.113$ \\
\hline IFN- $\gamma /$ IL-6 & $0.84 \pm 1.22$ & $0.21 \pm 0.19$ & $p=0.012$ \\
\hline \multicolumn{4}{|l|}{ Th1/Th2 } \\
\hline IL-2/IL-4 & $0.58 \pm 0.87$ & $1.46 \pm 1.76$ & $p=\mathbf{0 . 0 3 9}$ \\
\hline IL-2/IL-5 & $1.89 \pm 1.94$ & $1.35 \pm 1.71$ & $p=0.085$ \\
\hline IL-2/IL-10 & $0.92 \pm 1.35$ & $2.32 \pm 3.05$ & $p=0.182$ \\
\hline IL-2/IL-13 & $0.56 \pm 0.57$ & $0.17 \pm 0.15$ & $p<0.001$ \\
\hline TNF- $\alpha /$ IL-4 & $1.83 \pm 0.52$ & $13.17 \pm 20.77$ & $p=0.001$ \\
\hline TNF- $\alpha /$ IL-5 & $8.31 \pm 7.10$ & $12.96 \pm 19.77$ & $p=0.516$ \\
\hline TNF- $\alpha / I L-10$ & $2.84 \pm 0.92$ & $15.07 \pm 20.38$ & $p=0.002$ \\
\hline TNF- $\alpha /$ IL-13 & $2.25 \pm 0.94$ & $1.36 \pm 1.20$ & $p=0.011$ \\
\hline IFN- $\gamma /$ IL-4 & $0.83 \pm 0.16$ & $2.75 \pm 2.34$ & $p<0.001$ \\
\hline IFN- $\gamma /$ IL-5 & $3.72 \pm 3.57$ & $3.33 \pm 6.18$ & $p=0.802$ \\
\hline IFN- $\gamma /$ IL-10 & $1.27 \pm 0.25$ & $5.54 \pm 8.62$ & $p=0.042$ \\
\hline IFN- $\gamma /$ IL-13 & $1.01 \pm 0.29$ & $0.48 \pm 0.49$ & $p=0.005$ \\
\hline \multicolumn{4}{|l|}{ Th17/Th1 } \\
\hline IL-17A/IL-2 & $3.23 \pm 10.41$ & $8.27 \pm 8.97$ & $p<0.001$ \\
\hline IL-17A/TNF- $\alpha$ & $0.91 \pm 3.13$ & $0.84 \pm 0.73$ & $p=0.926$ \\
\hline IL-17A/IFN- $\gamma$ & $1.29 \pm 3.59$ & $4.30 \pm 5.89$ & $p=0.004$ \\
\hline IL-17F/IL-2 & $1.77 \pm 1.88$ & $2.14 \pm 2.05$ & $p=0.725$ \\
\hline IL-17F/TNF- $\alpha$ & $0.42 \pm 0.52$ & $0.26 \pm 0.24$ & $p=0.342$ \\
\hline IL-17F/IFN- $\gamma$ & $0.81 \pm 0.69$ & $0.70 \pm 0.63$ & $p=0.570$ \\
\hline \multicolumn{4}{|l|}{ Th17/Th2 } \\
\hline IL-17A/IL-4 & $1.34 \pm 4.36$ & $7.67 \pm 9.68$ & $p=0.020$ \\
\hline IL-17A/IL-5 & $2.01 \pm 3.17$ & $8.38 \pm 13.04$ & $p=0.064$ \\
\hline IL-17A/IL-10 & $1.66 \pm 4.39$ & $8.31 \pm 11.39$ & $p=0.034$ \\
\hline IL-17A/IL-13 & $0.79 \pm 1.19$ & $1.00 \pm 1.01$ & $p=0.555$ \\
\hline IL-17F/IL-4 & $0.68 \pm 0.72$ & $1.24 \pm 1.17$ & $p=0.070$ \\
\hline IL-17F/IL-5 & $1.84 \pm 1.35$ & $4.81 \pm 11.46$ & $p=0.302$ \\
\hline IL-17F/IL-10 & $1.07 \pm 0.93$ & $1.34 \pm 0.76$ & $p=0.340$ \\
\hline IL-17F/IL-13 & $0.77 \pm 0.64$ & $0.40 \pm 0.68$ & $p=0.084$ \\
\hline
\end{tabular}

Significant differences are given in bold, $p<0.05$

of controls aged under 60 years $(n=9)$ and those older than 60 years $(n=8)$ (Fig. $1 \mathrm{~d})$.

Our COVID-19 patients were mainly included in this study in the second week from the onset of disease (between 6 and 16th day). On the basis of data obtained from regular medical examinations during their hospitalization we analyzed the inflammatory parameters both before and after recruitment. Based on the lymphocyte counts and serum levels of CRP and IL-6, the patients were divided into the following two groups: those in whom inflammation worsened (7 patients: lymphocyte count decreased, while CRP and/or IL-6 levels increased)
Table 5 Mean values $(X \pm \mathrm{SD})$ of selected cytokine ratios in whole blood cultures from COVID-19 patients in whom the inflammatory process increased or decreased

\begin{tabular}{cccc}
\hline Cytokine index & COVID-19 patients & $p$ \\
\cline { 2 - 3 } & $\begin{array}{l}\text { Inflammation } \\
\text { increased }\end{array}$ & $\begin{array}{l}\text { Inflammation } \\
\text { decreased }\end{array}$ & \\
& $X \pm$ SD & & \\
\hline IL-17A/IL-6 & $0.37 \pm 0.43$ & $0.24 \pm 0.33$ & $p=0.492$ \\
IL-6/Th1 & & & \\
IL-6/IL-2 & $20.35 \pm 22.29$ & $6.24 \pm 5.21$ & $p=0.147$ \\
IL-6/IFN- $\boldsymbol{\gamma}$ & $10.34 \pm 9.23$ & $4.39 \pm 8.92$ & $p=0.089$ \\
IL-6/ TNF- $\alpha$ & $4.68 \pm 4.72$ & $1.88 \pm 2.88$ & $p=0.193$ \\
IL-17A/Th1 & & & \\
IL-17A/IL-2 & $8.47 \pm 18.62$ & $0.94 \pm 1.30$ & $p=0.103$ \\
IL-17A/IFN- $\boldsymbol{\gamma}$ & $\mathbf{3 . 2 6} \pm \mathbf{6 . 3 5}$ & $\mathbf{0 . 4 3} \pm \mathbf{0 . 4 9}$ & $\boldsymbol{p}=\mathbf{0 . 0 2 7}$ \\
IL-17A/TNF- $\alpha$ & $2.45 \pm 5.62$ & $0.23 \pm 0.33$ & $p=0.055$ \\
IL-17A/Th2 & & & \\
IL-17A/IL-4 & $\mathbf{3 . 6 2} \pm \mathbf{7 . 8 0}$ & $\mathbf{0 . 3 4} \pm \mathbf{0 . 3 8}$ & $\boldsymbol{p}=\mathbf{0 . 0 3 3}$ \\
IL-17A/IL-5 & $\mathbf{4 . 4 2} \pm \mathbf{5 . 1 1}$ & $\mathbf{0 . 9 5} \pm \mathbf{0 . 6 0}$ & $\boldsymbol{p}=\mathbf{0 . 0 1 8}$ \\
IL-17A/IL-10 & $3.97 \pm 7.72$ & $0.65 \pm 0.89$ & $p=0.299$ \\
IL-17A/IL-13 & $1.51 \pm 1.88$ & $0.47 \pm 0.57$ & $p=0.154$ \\
\hline Significant & & & \\
\hline
\end{tabular}

Significant differences are given in bold, $p<0.05$

and those in whom inflammation lessened (16 patients: lymphocyte count increased, CRP and/or IL-6 concentrations decreased). Comparison of cytokine values between the two groups showed statistically significant differences in IL$17 \mathrm{~A}(420.99 \pm 911.58$ vs $30.29 \pm 34.91, p=0.015)$ and IL-6 (915.13 \pm 826.23 vs $265.86 \pm 473.78, p=0.026$ ) levels (Fig. 2 ).

As there were statistically significant differences in the production of two cytokines (IL-17A and IL-6) between the two subgroups of COVID patients, we determined the IL-17A/IL-6 ratio as well as the ratios of IL-17A to type 1 (IL-2, IFN- $\gamma$ and TNF- $\alpha$ ) and type 2 cytokines (IL-4, IL-5, IL-10 and IL-13) and the ratio of IL- 6 to type 1 cytokines. Our results showed higher cytokine ratios in the subgroup of patients in whom inflammation increased, with statistically significant values for IL-17A/IFN- $\gamma$, IL-17A/IL-4 and IL-17A/IL-5 (Table 5).

To analyze a possible influence of methylprednisolone therapy on the cytokine production in vitro, a multivariate regression analysis was done. Our results show that there was no association of the duration of methylprednisolone therapy with the production of any cytokine tested. However, a negative impact of the applied dose of methyprednisolone on the production of several cytokines in vitro was shown, as follows: IL-17A ( $\beta=-0.458, p=0.048)$, IL-17F $(\beta=-0.498, p=0.038), \mathrm{IL}-22(\beta=-0.492, p=0.032)$ and IL-10 $(\beta=-0.454, p=0.050)$. 


\section{Discussion}

The aim of this study was to evaluate the cytokine producing ability of peripheral blood cells in severely ill COVID19 patients. Cytokine concentrations were measured in the supernatants of PHA-stimulated whole blood cultures in vitro. Our main results are as follows: (1) COVID-19 patients produce much smaller amounts of almost all tested cytokines than healthy control subjects even when the measured cytokine levels were recalculated for 1000 stimulated lymphocytes; (2) production of two cytokines (IL-6 and IL17A) was higher in COVID-19 patients in whom inflammation increased, leading to raised ratios of IL-17A to one type 1 (IFN- $\gamma$ ) and two type 2 (IL-4 and IL-5) cytokines, and (3) methylprednisolone applied in the therapy of COVID-19 patients had a negative impact on production of four cytokines (IL-17A, IL-17F, IL-22 and IL-10) in dosedependent manner.

First, we analyzed some hematological and biochemical parameters of severely ill COVID 19 patients and confirmed low lymphocyte numbers in our study group as observed by others. We found a negative correlation between lymphocyte counts and CRP levels in peripheral blood. Namely, greater inflammation was reflected by an increase of CRP concentration and the decrease in number of lymphocytes was more pronounced. Lymphopenia and perturbations of $\mathrm{T}$-cell homeostasis are very prominent features of severe COVID-19 [25-27].

Serious cases of COVID-19 have generally been associated with excessive cytokine release, particularly with large amounts of mainly proinflammatory types in plasma/serum samples. In the most severe cases, hyperproduction of IL-1 $\beta$, IL-2, IL-6, IL-12, IFN- $\gamma$ and TNF- $\alpha$ was documented, which preferentially target lung tissue [13, 28]. Increased levels of proinflammatory Th17 cytokines $[3,14]$ and Th 2 cytokines (IL-4 and IL-10) [15] were also observed. For the pathogenesis of disease, enhanced secretion of IL-6 [29, 30] and diminished secretion of interferons $[31,32]$ are of particular importance.

Our results showed that PHA-stimulated whole blood cells from severely ill COVID-19 patients produce several-fold less cytokines than control cells. Given that we did not measure concentrations of cytokines in supernatants obtained from unstimulated whole blood cultures of COVID-19 patients and control subjects, we cannot exclude the possibility that at least a part of cytokines measured in this study was released independently from PHA-stimulation. But, we do not believe that this significantly affects the results presented here since the whole blood cultures from control subjects produce a much higher levels of cytokines than blood cells from COVID-19 patients when the both stimulated with PHA in vitro. In another in vitro study [18], it was documented that the levels of cytokines in unstimulated cultures of COVID-19 patients, and especially controls were undetectable or very low.

Since COVID-19 patients expressed varying degrees of lymphopenia, the numbers of stimulated lymphocytes differed individually among both patients and control subjects, so the measured cytokine values were recalculated in relation to 1000 stimulated lymphocytes in all subjects. Again, we found significantly lower cytokine levels in the culture media obtained from COVID-19 patients compared with media from the healthy subjects, except for three cytokines (IL-4, IL-9 and IL-10). The lack of significant differences for production of these cytokines between the patient and control groups might be a consequence of the small sample size and high individual variation within the groups. Namely, as earlier shown for lipopolysaccharide (LPS)-stimulated cytokine production ex vivo [33], PHA-stimulated cytokine production in our whole blood cultures from the COVID-19 patients and control subjects varied greatly from individual to individual.

On the basis of our results, we can conclude that COVID19 patients have a diminished ability to produce cytokines in response to strong and non-specific PHA stimulation in vitro. This is consistent with the findings of most studies examining the response of blood cells from COVID-19 patients to non-specific in vitro stimulation. Thus, in one investigation, peripheral blood mononuclear cells were stimulated by overnight incubation with anti-CD3/anti-CD28 antibodies to activate $\mathrm{T}$ cells or with LPS to activate monocytes. The IFN- $\gamma$-producing T cells and TNF- $\alpha$-producing monocytes were quantified using an ELISpot functional immunoassay [20] which showed that mononuclear cells from COVID-19 patients produced less TNF- $\alpha$ and IFN-y than controls. Ex vivo stimulation with anti-CD3/anti-CD28 antibodies was used by de Biasi et al. [14], who observed increased amounts of IL-2, IL-17, TNF- $\alpha$ and IFN- $\gamma$ in the cytoplasm of T cells of COVID-19 patients when compared to controls. In another study the levels of type I and type II interferons (IFN) were determined after in vitro stimulation of immune cells from COVID-19 patients with Tolllike receptor 7/8 (TLR 7/8) agonist and anti-CD3 antibody [18]. Reduced levels of both types of interferon were found that correlated with increase in disease severity. A different model was used to assess the activation of lymphocyte from COVID-19 patients [7] in which blast cell formation was examined after in vitro activation of whole blood with mitogens (pokeweed and Concanavalin A), Staphylococcal super-antigens (SEA/SEB), and common viral antigens (adenovirus, cytomegalovirus, Herpes simplex virus 1, Herpes simplex virus 2 and Varicella zoster virus). In patients with severe COVID-19 reduced blast formation upon stimulation with the selected viral antigens or Concanavalin A was observed, but not after stimulation with pokeweed mitogen 
or Staphylococcal super-antigens. With a few exceptions, most studies have shown very poor responses of blood cells from COVID patients to non-specific in vitro stimulation, as found here. Some differences in the findings cited might be a consequence of diversity in study design, the use of various stimulating agents and/or times of incubation.

The in vitro study of Avendaño-Ortiz et al. [34] is interesting. They showed that antigen-specific stimulation of T-cells proliferation was significantly reduced by SARS$\mathrm{CoV}-2$ proteins (S glycoprotein, $\mathrm{N}$ protein and papain/like protease) after 5 days' stimulation indicating that patients with long-term SARS-CoV-2 infection might induce a specific monocyte profile that exhibits a significant blockade of $\mathrm{T}$-cell proliferation and function. Mechanisms leading to a weakened response of peripheral blood cells from COVID19 patients in vitro could be due to blood cell exhaustion $[14,18]$, or active suppression during the immune response to SARS-CoV-2 [20]. Both hypotheses are supported by the results of studies published so far [20,35].

Anti-inflammatory therapies, including glucocorticosteroids, would play a protective role in severely ill COVID19 patients [36]. Corticosteroids suppress inflammation by reducing the number and/or activation of lymphocytes and excessive production of inflammatory mediators, cytokines and chemokines [37, 38]. Thus, CD4+ lymphocytes were significantly lower in patients treated with dexamethasone compared to those who were not [39]. In septic shock, lowdose hydrocortisone was reported to prevent release of proinflammatory cytokines [40]. In a recently published study it was shown that dexamethasone might shorten the time between the start of invasive mechanical ventilation and the occurrence of ventilator-associated pneumonia, suggesting a possible worsening of COVID-19-induced immune dysfunction with this treatment [39]. Methylprednisolone treatment was applied in all COVID-19 patients enrolled in this study, since all patients had viral pneumonia with increased values of inflammatory parameters, requiring oxygen treatment. Although the corticosteroid therapy is recommended for severely ill patients with COVID-19 [41], our study shows that the methylprednisolone used in the treatment of patients with COVID-19 inhibits the production of several cytokines in dose dependent manner in vitro.

Given that the large individual variation in production of cytokines was not lost after correction for cell number and that in the both study groups some subjects produced more cytokines than others, we analyzed the relationships between selected cytokines for each subject individually. The ratios between T helper 1 (Th1) cytokines (IL-2, TNF- $\alpha$ IFN- $\gamma$ ) and IL-13 (a Th2 cytokine) were higher in COVID-19 patients than in the controls. However, the ratios of Th1 cytokines with IL-4, which also belongs to the Th2 type of cytokines, were lower in COVID-19 patients than in control subjects. Ratios for IL-2, TNF- $\alpha$ and IFN- $\gamma$ with
IL-6 were generally higher in COVID-19 patients than in controls. The ratio of two cytokines might be greater if production of one is increased and/or production of the other is decreased. Although cytokines that mainly belong to the Th1/Th17 types are included in the pathogenesis of COVID19 infection [14], a clear shift to Th1 type cytokines was not seen in our study group but an effect of corticosteroid drugs on Th1/Th2 ratios cannot be excluded [42]. The ratios of some Th17/Th1 cytokines (IL-17A/IL-2, IL-17A/IFN- $\gamma$ ) and Th17/Th2 cytokines (IL-17A/IL-4, IL-17A/IL-10) were lower in COVID-19 patients than in controls. Thus, when we analyzed our entire group of COVID-19 patients a tendency towards production of Th17 cytokines was not observed, which is contrary to the results of others [14].

Concerning possible associations of cytokine production with gender and age in severely ill COVID-19 patients, we showed that the mean concentrations of almost all cytokines tended to be higher in males than in females, but significantly only for IL-13. Since the start of the COVID-19 epidemic, it has been evident that among seriously ill patients there were more men than women [43]. However, little is known regarding IL-13 concentration in COVID-19 patients. Huang et al. found no difference in serum IL-13 levels between those requiring admission to an Intensive Care Unit (ICU) and those who did not [43], while a directly proportional association between IL-13 levels and the viral load of SARS-CoV-2 was observed in another study [13]. IL-13 is a type 2 cytokine, known to induce production of other Th2 cytokines in the immune response to parasitic infections and hypersensitivity reactions [13]. Hypothetically, production of IL-13 might stimulate Th2 cells to synthesize interleukin 10, a contraregulatory cytokine that suppresses the Th1 immune response [13].

Patients of advanced age and those with underlying conditions (e.g. obesity, hypertension, chronic obstructive pulmonary disease, diabetes, and/or cardiovascular disease, etc.) are at higher risk of disease progression, often resulting in an unfavorable outcome [24, 29, 35]. Studies examining the activity of the immune system during aging indicate the following two basic mechanisms that can alter the immune response: immunosenescence and inflamm-aging [44]. We found no statistically significant differences in cytokine levels between the subgroup of patients aged under 60 years and those older than 60 years. There was no correlation of cytokine levels with the age of patients, except for interleukin 22 (IL-22). IL-22 is produced by Th22 cells and is known to play a tissue protective role in inflammation [45].

The relatively long period for obtaining of blood samples from COVID-19 patients includes the time of intensification of inflammation and the possible development of lymphocyte exhaustion/immunosupression [46]. Since the rate of disease progression is individual and unpredictable, we subsequently (on the basis of available medical documentation during 
hospitalization) analyzed inflammatory markers before and after the days of sampling. According to serum levels of CRP, IL-6 and lymphocyte numbers in peripheral blood, the patients were divided into the following two groups: one in which inflammatory parameters were raised and the other in which inflammation had already begun to decrease. Comparison between the two groups showed statistically significant differences in IL-6 and IL-17A production.

An elevated level of interleukin-6 is designated as a red flag of systemic inflammation with a poor prognosis in COVID-19 patients [15, 47, 48]. High expression of IL-6 in patients with COVID-19 can accelerate the inflammatory process, contributing to a cytokine storm [15]. In the subgroup of COVID-19 patients with raised inflammatory mediators, in vitro production of IL-6 was greater than in those in whom inflammation lessened. That led to lower Th1/ IL-6 cytokine ratios, but the differences between the two subgroups of patients did not reach statistical significance. IL-6 has negative effects on Th1 differentiation, while in joint action with some other cytokines (IL- $1 \beta$ and TGF- $\beta$ ) it induces Th17 subset differentiation [3]. IL-17 is a proinflammatory cytokine that plays a role in tissue damage, physiological stress, and infection [15] and can enhance inflammation and activate neutrophils [14]. Some studies showed an elevated level of IL-17 in COVID-19 patients during a cytokine storm [29], but others found normal levels of IL-17 regardless of the severity of COVID-19 [49]. In this in vitro study we detected greater production IL-17A when inflammation was augmented, which led to higher Th17/Th2 cytokine ratios than in patients in the resolving phase of the disease, especially significant for IL-17A/IL-4 and IL-17A/ IL-5. Also, cytokine ratios for IL-17A with Th1 cytokines were slightly higher during the phase of intensification of the inflammatory process, which was statistically significant for the IL-17A/IFN- $\gamma$ ratio. Taken together, the increase in production of two proinflammatory cytokines (IL-6 and IL-17) in the early stages of inflammation noted here is consistent with earlier findings showing elevated levels of these cytokines in severely ill [14] and critically ill [17] COVID-19 patients. This indicates that IL-6 overproduction and infiltration of Th17 cells play important roles in acute lung injury during ARDS. Since some patients in our study group were included while inflammation increased and others when it was subsiding, our findings indicate that the stage of the inflammatory process might influence the results obtained.

Other studies in which in vitro stimulation with PHA was performed have shown that both Th1 and Th2 cytokines were reduced in adult and child patients with sepsis and nosocomial infections in vitro [50-52]. Cavaillon [52] suggested that this is against the dogma claiming that sepsis is associated with up-regulation of the Th2 response and down-regulation of the Th1 response. In patients with sepsis and septic shock cytokine down-regulation was transient and normal production of cytokines from T cells was restored after 7 days. Our results suggest that in COVID-19 patients there is a prolonged decrease of cytokine production given that they were included in the study from 6 to 16th day after onset of the disease. Moreover, down-regulation of cytokine production was not limited to Th1 and Th2 cytokines, because we showed very pronounced decreases in production of $\mathrm{Th} 1 / \mathrm{Th} 2 / \mathrm{Th} 9 /$ Th17/Th22 cytokines, which was not caused by the smaller number of stimulated cells. Also, this markedly reduced cytokine production in patients with COVID-19 was accompanied by an imbalance in production between Th17 and Th2, and to some extent Th17 and Th1 cytokines, in the phase of intensification of the inflammatory process. However, our results obtained by nonspecific in vitro stimulation of whole blood cells do not have to be consistent with the abilities of T lymphocytes in infected tissues. Namely, tissue derived cells are either fully responsive to ex vivo stimuli or even primed, in contrast to cells derived from blood [19]. Therefore, the hyporesponsiveness of circulating leukocytes is considered to be a marker of immune depression and may explain the enhanced sensitivity of these patients to nosocomial infections [19]. In the cited review paper Cavaillon postulated that SIRS and the compensatory anti-inflammatory response syndrome (CARS) most probably occur concomitantly in different compartments, whereby SIRS predominates within the inflamed tissues while blood leukocytes show hyporeactivity. Moreover, these differences might be a normal reaction of the immune system to prevent systemic inflammation when an inflammatory process exists locally in some tissues [53].

To the best of our knowledge, this is a first study investigated cytokine producing ability of blood cells from COVID-19 patients stimulated with a very powerful $\mathrm{T}$ cell mitogen, PHA. The results obtained here mainly correlate with the results of other studies using nonspecific in vitro stimulators. We believe that the decreased ability of blood cells to produce cytokines during an in vitro stimulation deserves attention, despite the fact that there is no correlation between the levels of IL- 6 in serum and supernatant samples from the same patient. The levels of cytokines in serum samples might reflect the production of cytokines in vivo, released from the site of inflammation and/or from blood cells in blood vessels. On the other hand, cytokine concentrations measured in culture medium after in vitro stimulation represents the ability of blood cells to respond by cytokine production, if necessary.

In conclusion, we could say that peripheral blood cells from severely ill COVID-19 patients have a much reduced ability to produce Th1/Th2/Th9/Th17/Th22 cytokines after strong non-specific in vitro stimulation with PHA. When inflammation was intensified, blood cells produced more IL-6 and IL-17, which led to increases of some Th17/Th1 and Th17/Th2 ratios, skewing towards the Th17 type of response. The methylprednisolone used in the treatment of 
patients with COVID-19 influences the production of several cytokines in dose dependent manner. Our results indicate that the stage of the inflammatory process at the time of sampling and the dose of glucocorticosteroids applied in the therapy of COVID-19 patients might influence cytokine production upon non-specific stimulation of $\mathrm{T}$ cells in vitro.

Acknowledgements The study was supported by the Ministry of Education, Science and Technological Development of the Republic of Serbia (Grant nos. III41010 and ON175069).

\section{Declarations}

Conflict of interest The authors stated that there are no conflicts of interest regarding the publication of this article.

\section{References}

1. Wu Z, McGoogan JM. Characteristics of and important lessons from the coronavirus disease 2019 (COVID-19) outbreak in China: summary of a report of 72314 cases from the Chinese center for disease control and prevention. JAMA. 2020;323:1239-42.

2. Ye Q, Wang B, Mao J. The pathogenesis and treatment of the 'Cytokine Storm' in COVID-19. J Infect. 2020;80:607-13.

3. Pat J, Alkan SS. How does SARS-CoV-2 manipulates the immune system? Critical role of Th1, Th2, Th17 responses. Turk J Immunol. 2020;8(2):100-2.

4. Matic S, Popovic S, Djurdjevic P, Todorovic D, Djordjevic N, Mijailovic Z, Sazdanovic P, Milovanovic D, Zecevic DR, Petrovic M, Sazdanovic M, Zornic N, Vukicevic V, Petrovic I, Matic S, Baskic D. SARS-CoV-2 infection induces mixed M1/M2 phenotype in circulating monocytes and alterations in both dendritic cell and monocyte subsets. PLoS ONE. 2020;15(12):e0241097. https://doi.org/10.1371/journal.pone.0241097.

5. Ahmed SF, Quadeer AA, McKay MR. Preliminary identification of potential vaccine targets for the COVID-19 coronavirus (SARSCoV-2) based on SARS-CoV immunological studies. Viruses. 2020;12(3):254-73.

6. Qin CC, Zhou LL, Hu Z, Zhang S, Yang S, Tao Y, Xie C, Ma K, Shang K, Wang W, Tian D-S. Dysregulation of immune response in patients with coronavirus 2019 (COVID-19) in Wuhan, China. Clin Infect Dis. 2020;71:762-8.

7. Adamo S, Chevrier S, Cervia C, Zurbuchen Y, Raeber ME, Yang L, Sivapatham S, Jacobs A, Baechli E, Rudiger A, Stüssi-Helbling M, Huber LC, Schaer DJ, Bodenmiller B, Boyman O, Nilsson J. Profound dysregulation of T cell homeostasis and function in patients with severe COVID-19. Allergy. 2021;76(9):2866-81.

8. Mohamed Khosroshahi L, Rokni M, Mokhtari T, Noorbakhsh F. Immunology, immunopathogenesis and immunotherapeutics of COVID-19; an overview. Int Immunopharmacol. 2021;93:107364. https://doi.org/10.1016/j.intimp.2020.107364 (Epub 2021 Jan 5. PMID: 33486333; PMCID: PMC7784533).

9. Shah VK, Firmal P, Alam A, Ganguly D, Chattopadhyay S. Overview of immune response during SARS-CoV-2 infection: lessons from the past. Front Immunol. 2020;11:1949. https://doi.org/10. 3389/fimmu.2020.01949.

10. Jung HE, Lee HK. Current understanding of the innate control of toll-like receptors in response to SARS-CoV-2 infection. Viruses. 2021;13:2132. https://doi.org/10.3390/v13112132.
11. Astuti I, Ysrafil I. Severe acute respiratory syndrome coronavirus 2 (SARS-CoV-2): an overview of viral structure and host response. Diabetes Metab Syndr. 2020;14(4):407-12. https://doi. org/10.1016/j.dsx.2020.04.020.

12. Pons MJ, Ymaña B, Mayanga-Herrera A, Sáenz Y, AlvarezErviti L, Tapia-Rojas S, Gamarra R, Blanco AB, Moncunill G, Ugarte-Gil MF. Cytokine profiles associated with worse prognosis in a hospitalized peruvian COVID-19 cohort. Front Immunol. 2021;12: 700921. https://doi.org/10.3389/fimmu.2021.700921.

13. Costela-Ruiz VJ, Illescas-Montes R, Puerta-Puerta JM, Ruiz C, Melguizo-Rodriguez L. SARS-CoV-2 infection: the role of cytokines in COVID-19 disease. Cytokine Growth Factor Rev. 2020;54:62-75.

14. De Biasi S, Meschiari M, Gibellini L, Bellinazzi C, Borella R, Fidanza L, Gozzi L, Iannone A, Tartaro DL, Mattioli M, Paolini A, Menozzi M, Milić J, Franceschi G, Fantini R, Tonelli R, Sita M, Sarti M, Trenti T, Brugioni L, Cicchetti L, Facchinetti F, Pietrangelo A, Clini E, Girardis M, Guaraldi G, Mussini C, Cossarizza A. Marked T cell activation, senescence, exhaustion and skewing towards TH17 in patients. Nat Commun. 2020;11:3434. https://doi.org/10.1038/s41467-020-17292-4.

15. Hirawat R, Saifi MA, Godugu C. Targeting inflammatory cytokine storm to fight against COVID-19 associated severe complications. Life Sci. 2021;267:11892328.

16. Song P, Li W, Xie J, Hou Y, You C. Cytokine storm induced by SARS-CoV-2. Clin Chim Acta. 2020;509:280-7. https://doi.org/ 10.1016/j.cca.2020.06.017.

17. Pasrija R, Naime M. The deregulated immune reaction and cytokines release storm (CRS) in COVID-19 disease. Int Immunopharmacol. 2021;90:107225.

18. Ruetsch C, Brglez V, Crémoni M, Zorzi K, Fernandez C, BoyerSuavet S, Benzaken S, Demonchy E, Risso K, Courjon J, Cua E, Ichai C, Dellamonica J, Passeron T, Seitz-Polski B. Functional exhaustion of type I and II interferons production in severe COVID-19 patients. Front Med. 2021;7: 603961. https://doi.org/ 10.3389/fmed.2020.603961.

19. Cavaillon J-M, Adib-Conquy M, Cloëz-Tayarani I, Fitting C. Immunodepression in sepsis and SIRS assessed by ex vivo cytokine production is not a generalized phenomenon: a review. $\mathrm{J}$ Endotoxin Res. 2021;7(2):85-93.

20. Remy KE, Mazer M, Striker DA, Ellebedy AH, Walton AH, Unsinger J, Blood TM, Mudd PA, Yi DJ, Mannion DA, Osborne DF, Martin RS, Anand NJ, Bosanquet JP, Blood J, Drewry AM, Caldwell CC, Turnbull IR, Brakenridge SC, Moldwawer LL, Hotchkiss RS. Severe immunosuppression and not a cytokine storm characterizes COVID-19 infections. JCI Insight. 2020;5(17): e140329. https://doi.org/10.1172/jci.insight.140329.

21. Ceuppens JL, Baroja ML, Lorre K, Van Damme J, Billiau A. Human T cell activation with phytohemagglutinin. The function of IL-6 as an accessory signal. J Immunol. 1988;141(11):3868-74.

22. Organization WH. Clinical management of COVID-19: interim guidance, 27 May 2020. World Health Organization; 2020. Laboratory testing strategy recommendations for COVID-19. Interim guidance. 21 March 2020. Geneva, World Health Organization, 2020. WHO/2019-nCoV/clinical/2020.5.

23. Simonovic SZ, Mihaljevic O, Majstorovic I, Djurdjevic P, Kostic I, Djordjevic OM, Teodorovic LM. Cytokine production in peripheral blood cells of patients with differentiated thyroid cancer: elevated $\mathrm{Th} 2 / \mathrm{Th} 9$ production before and reduced Th 2 cytokine production after radioactive iodine therapy. Cancer Immunol Immunother. 2015;64:75-82.

24. Zivancevic-Simonovic S, Mihaljevic O, Majstorovic I, Popovic S, Markovic S, Milosevic-Djordjevic O, Jovanovic Z, Mijatovic-Teodorovic L, Mihajlovic D, Colic M. Cytokine production in patients with papillary thyroid cancer and associated autoimmune Hashimoto thyroiditis. Cancer Immunol Immunother. 2015;64:1011-9. 
25. Wang J, Jiang M, Chen X, Montaner LJ. Cytokine storm and leukocyte changes in mild versus severe SARS-CoV-2 infection: review of 3939 COVID-19 patients in China and emerging pathogenesis and therapy concepts. J Leukoc Biol. 2020;108(1):17-41. https://doi.org/10.1002/JLB.3COVR0520-272R (Epub 2020 Jun 13).

26. Diao B, Wang C, Tan Y, Chen X, Liu Y, Ning L, Chen L, Li M, Liu Y, Wang G, Yuan Z, Feng Z, Wu Y, Chen Y. Reduction and functional exhaustion of $\mathrm{T}$ cells in patients with coronavirus disease 2019 (COVID-19). Front Immunol. 2020;11:827-41.

27. Bodenmiller B, Boyman O, Nilsson J. Profound dysregulation of T cell homeostasis and function in patients with severe COVID-19. Allergy. 2021;76:2866-81.

28. Fraser DD, Cepinskas G, Slessarev M, Martin C, Daley M, Miller MR, O'Gorman DB, Gill SE, Patterson EK, dos Santos CC, On behalf of the Lawson COVID-19 Study Team. Inflammation profiling of critically ill coronavirus disease 2019 patients. Crit Care Explor. 2020;2(6):e0144. https://doi.org/10.1097/cce.0000000000 000144.

29. Huang L, Zhao X, Qi Y, Li H, Ye G, Liu Y, Zhang Y, Gou J. Sepsis-associated severe interleukin-6 storm in critical coronavirus disease 2019. Cell Mol Immunol. 2020;17(10):1092-4. https:// doi.org/10.1038/s41423-020-00522-6.

30. Copaescu A, Smibert O, Gibson A, Phillips EJ, Trubiano JA. The role of IL- 6 and other mediators in the cytokine storm associated with SARS-CoV-2 infection. J Allergy Clin Immunol. 2020;146(3):518-34.e1. https://doi.org/10.1016/j.jaci.2020.07. 001 .

31. Hadjadj J, Yatim N, Barnabei L, Corneau A, Boussier J, Smith N, Péré H, Charbit B, Bondet V, Chenevier-Gobeaux C, Breillat P, Carlier N, Gauzit R, Morbieu C, Pène F, Marin N, Roche N, Szwebel TA, Merkling SH, Treluyer JM, Veyer D, Mouthon L, Blanc C, Tharaux PL, Rozenberg F, Fischer A, Duffy D, Rieux-Laucat F, Kernéis S, Terrier B. Impaired type I interferon activity and exacerbated inflammatory responses in severe Covid-19 patients. Science. 2020;369(6504):718-24.

32. Wang Z, Pan H, Jiang B. Type I IFN deficiency: an immunological characteristic of severe COVID-19 patients. Signal Transduct Target Ther. 2020;5(1):198. https://doi.org/10.1038/ s41392-020-00306-4.

33. Wurfel MM, Park WY, Radella F, Ruzinski J, Sandstrom A, Strout J, Bumgarner RE, Martin TR. Identification of high and low responders to lipopolysaccharide in normal subjects: an unbiased approach to identify modulators of innate immunity. J Immunol. 2005;175:2570-8.

34. Avendaño-Ortiz J, Lozano-Rodríguez R, Martín-Quirós A, Maroun-Eid C, Terrón V, Valentín J, Montalbán-Hernández K, de la Bastida FR, García-Garrido MA, Cubillos-Zapata C, Del Balzo-Castillo Á, Aguirre LA, López-Collazo E. Proteins from SARS-CoV-2 reduce T cell proliferation: a mirror image of sepsis. Heliyon. 2020;6(12): e05635. https://doi.org/10.1016/j.heliyon. 2020.e05635.

35. Bordallo B, Bellas M, Cortez AF, Vieira M, Pinheiro M. Severe COVID-19: what have we learned with the immunopathogenesis? Adv Rheumatol. 2020;60:50-62.

36. Sherwani S, Khan MWA. Cytokine response in SARS-CoV-2 infection in the elderly. J Inflamm Res. 2020;13:737-47. https:// doi.org/10.2147/JIR.S276091 (Published 2020 Oct 20).

37. Ni YN, Chen G, Sun J, Liang BM, Liang ZA. The effect of corticosteroids on mortality of patients with influenza pneumonia: a systematic review and meta-analysis. Crit Care. 2019;23(1):99.

38. Langarizadeh MA, RanjbarTavakoli M, Abiri A, Ghasempour A, Rezaei M, Ameri A. A review on function and side effects of systemic corticosteroids used in high-grade COVID-19 to prevent cytokine storms. EXCLI J. 2021;20:339-65. https://doi.org/10. 17179/excli2020-3196.PMID:33746666;PMCID:PMC7975631.
39. Cour M, Simon M, Argaud L, Monneret G, Venet F. Effects of dexamethasone on immune dysfunction and ventilator-associated pneumonia in COVID-19 acute respiratory distress syndrome: an observational study. J Intensive Care. 2021;9(1):1-4.

40. Keh D, Boehnke T, Weber-Cartens S, Schulz C, Ahlers O, Bercker S, et al. Immunologic and hemodynamic effects of "lowdose" hydrocortisone in septic shock: a double-blind, randomized, placebo-controlled, crossover study. Am J Respir Crit Care Med. 2003;167:512-20. https://doi.org/10.1164/rccm.200205-446OC.

41. Zhang W, Zhao Y, Zhang F, Wang Q, Li T, Liu Z, Wang J, Qin Y, Zhang X, Yan X, Zeng X, Zhang S. The use of anti-inflammatory drugs in the treatment of people with severe coronavirus disease 2019 (COVID-19): the perspectives of clinical immunologists from China. Clin Immunol. 2020;214:108393-108393.

42. Elenkov IJ. Glucocorticoids and the Th1/Th2 balance. Ann N Y Acad Sci. 2004;1024:138-46.

43. Huang C, Wang Y, Li X, Ren L, Zhao J, Hu Y, Zhang L, Fan G, Xu J, Gu X, Cheng Z, Yu T, Xia J, Wei Y, Wu W, Xie X, Yin W, Li H, Liu M, Xiao Y, Gao H, Guo L, Xie J, Wang G, Jiang R, Gao Z, Jin Q, Wang J, Cao B. Clinical features of patients infected with 2019 novel coronavirus in Wuhan, China. Lancet. 2020;395:497506. https://doi.org/10.1016/S0140-6736(20)30183-5).

44. Bajaj V, Gadi N, Spihlman AP, Wu SC, Choi CH, Moulton VR. Aging, immunity, and COVID-19: How age influences the host immune response to coronavirus infections? Front Physiol. 2021;11:pN.PAG-N.PAG.

45. Akdis M, Palomares O, van de Veen W, van Splunter M, Akdis CA. TH17 and TH22 cells: a confusion of antimicrobial response with tissue inflammation versus protection. J Allergy Clin Immunol. 2012;129:1438-49.

46. Lopez-Collazo E, Avendano-Ortiz J, Martin-Quiros A, Aguirre LA. Immune response and COVID-19. A mirror image of sepsis. Int J Biol Sci. 2020;16:2479-89.

47. Zhou F, Yu T, Du R, Fan G, Liu Y, Liu Z, Xiang J, Wang Y, Song B, Gu X, Guan L, Wei Y, Li H, Wu X, Xu J, Tu S, Zhang Y, Chen $\mathrm{H}, \mathrm{Cao}$ B. Clinical course and risk factors for mortality of adult in patients with COVID-19 in Wuhan, China: a retrospective cohort study. Lancet. 2020;395:1054-62. https://doi.org/10.1016/S01406736(20)30566-3.

48. Melo AKG, Milby KM, Caparroz ALMA, Pinto ACPN, Santos RRP, Rocha AP, Ferreira GA, Souza VA, Valadares LDA, Vieira RMRA, Pileggi GS, Trevisani VFM. Biomarkers of cytokine storm as red flags for severe and fatal COVID-19 cases: a living systematic review and meta-analysis. PLoS ONE. 2021;16(6):121. https://doi.org/10.1371/journal.pone.0253894.

49. Wan S, Yi Q, Fan S, Lv J, Zhang X, Guo L, Lang C, Xiao Q, Xiao K, Yi Z, Qiang M, Xiang J, Zhang B, Chen Y. Characteristics of lymphocyte subsets and cytokines in peripheral blood of 123 hospitalized patients with 2019 novel coronavirus pneumonia (NCP). MedRxiv. 2020. https://doi.org/10.1101/2020.02.10.20021832.

50. Muret J, Marie C, Fitting C, Payen D, Cavaillon J-M. Ex vivo T-lymphocyte derived cytokine production in SIRS patients is influenced by experimental procedures. Shock. 2020;13:169-74.

51. De AK, Kodys KM, Pellegrini J, Yeh B, Furse RK, Bankey P, Miller-Graziano CL. Induction of global anergy rather than inhibitory Th2 lymphokines mediates post-trauma T-cell immunodepression. Clin Immunol. 2000;96:52-66.

52. Cavaillon J-M. Altered immune status of circulating T lymphocytes during sepsis: children also. Crit Care. 2014;18:486-7.

53. Munford RS, Pugin J. Normal response to injury prevent systemic inflammation and can be mmunosuppressive. Am J Respir Crit Care Med. 2001;163:316-21.

Publisher's Note Springer Nature remains neutral with regard to jurisdictional claims in published maps and institutional affiliations. 